\title{
Best Practices Utilized by The EV Project for Non- Residential Alternating Current Level 2 Electric Vehicle Supply Equipment Infrastructure Planning
}

Idaho National Laboratory

June 2015

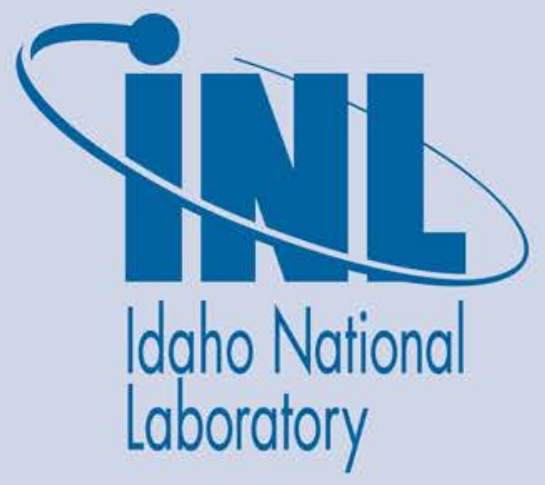

The INL is a U.S. Department of Energy National Laboratory operated by Battelle Energy Alliance 


\section{Best Practices Utilized by The EV Project for Non-Residential Alternating Current Level 2 Electric Vehicle Supply Equipment Infrastructure Planning}

Idaho National Laboratory

June 2015

Idaho National Laboratory
Idaho Falls, Idaho 83415

http://www.inl.gov

Prepared for the

U.S. Department of Energy

Assistant Secretary for __, OR Office of Under DOE Idaho Operations Office

Contract DE-AC07-05ID14517 


\section{Best Practices Utilized by The EV Project for Non-Residential Alternating Current Level 2 Electric Vehicle Supply Equipment Infrastructure Planning}

June 2015

\section{Key Conclusions}

- Key considerations for deployment of publicly accessible alternating current (AC) Level 2 electric vehicle supply equipment (EVSE) infrastructure should include expected high utilization and high turnover of vehicles charging at these EVSE.

- The objective in gaining highly utilized EVSE is best met by advanced detailed planning similar to the criteria employed by The EV Project.

- The selection of venue carries significant importance in identifying locations where PEV drivers are likely to park and, thus, charge when convenient or needed.

\section{Introduction}

The lack of public charging infrastructure for plug-in electric vehicles (PEVs) has been identified as a barrier to their widespread adoption. Federal and state grants have been awarded to promote public charging and encourage retail businesses to install charging infrastructure. A common question for charger installations is, "Where should the chargers be placed?" One of the objectives of The EV Project was to study the interaction of PEV drivers with public infrastructure; therefore, The EV Project attempted to develop a method for determine where EVSE should be placed.

In the early stages of PEV sales in local markets, the options for siting publicly accessible EVSE were as follows:

1. Plan locations associated with key attraction sites where PEV parking is anticipated

2. Directly solicit retail and public charging hosts for widespread placement

3. Ask PEV drivers who were early adopters where they want public infrastructure

4. Identify sites near known high-traffic areas.

The EV Project chose the first approach for planned deployment. This planning process, trademarked EV Micro-
Climate $^{\circledR}$, was used in the first five regions of The EV Project and was documented and evaluated. ${ }^{1}$

All four approaches were addressed in separate reports involving a case study of San Diego. Application of the EV Micro-Climate planning efforts in San Diego is reported in Reference 2. The second approach identified was employed by others and, to a lesser degree, by some of the regions of The EV Project discussed in Reference 3. The third approach was employed in the San Diego region through interviews and surveys and the results were explored in References 4 and 5 . The fourth approach was also addressed in Reference 3. Further analysis of where PEV drivers park in the San Diego region was provided in Reference 6.

This paper synthesizes the previous work to identify the best practices in locating AC Level 2 PEV charging infrastructure using San Diego as the case study.

\section{Background}

The EV Project was designed as an infrastructure study. It was designed to generate a wealth of directly applicable technical and professional experience for jumpstarting regional PEV adoption and replicating business models that lead to sustainable, market-based deployment of charging infrastructure. Charging opportunities away from home increase consumer confidence that they can return home before fully depleting their battery along the way and becoming stranded. The counter to this "range anxiety" is the range confidence provided by an abundance of available EVSE in locations where the driver is likely to park during travel. The availability and use of public charging infrastructure is also an incentive for growth in adoption of PEVs. Appendix Figure A-1 was used by The EV Project's regional advisory groups to illustrate this growth.

The typical publicly available EVSE in the 1990s was AC Level 2 EVSE. The challenge at that time was to find commercial hosts who would participate in the cost of EVSE and its installation, with an ability to collect revenue for use of the EVSE. Consequently, the strategy for locating units was to find willing charging site hosts. Often the locations did not match locations where the PEV would normally drive; however, the early enthusiasts were willing to accept that inconvenience. It was not a sustainable model for widespread adoption.

It is important that the location of public EVSE be planned to be in the most effective and visible location; therefore, it will be available to and used by PEV drivers. In addition, it needs to satisfy the charging site host or EVSE provider with a financial benefit to off-set the cost of the equipment 
and its installation. The purpose of this paper is to identify the best practices in deploying this pubic charging infrastructure.

\section{Key Considerations}

The EV Project assumed there would be competing interests in identifying sites for public infrastructure once the advisory groups assisted in site planning and selection processes were established. For example, some regions had high interest in locating EVSE in public or municipal locations because of their visibility or because they supported local political agendas. To maintain the focus of the planning process, The EV Project provided specific guidance to each of the regions on priorities for EVSE siting. This guidance included the following:

1. The site must be within The EV Project's study boundaries to meet the data and analysis requirements.

2. The site must be a location where PEVs enrolled in The EV Project would be likely to charge.

3. Site selection should favor those areas where EVSE are easily accessible throughout the day and evening and weekday and weekend.

4. Some EVSE should be placed in areas to support special studies and specific data collection activities.

All criteria were designed to place EVSE in locations expected to be frequented by PEVs enrolled in The EV Project and those outside the project. Criteria 2 and 3 specifically focused on high utilization and high turnover of PEVs. Because these attributes also factor into a financial benefit for the charging site host or EVSE provider, they support one of the objectives of creating a sustainable business model.

It was also thought that an unused EVSE, regardless of location, would be a disincentive to adoption of PEVs. Public observance of an unused EVSE may create the impression that PEVs are not being accepted by the public. In addition, unused EVSE in desirable parking locations marked for charging only can create a negative impression for non-PEV drivers looking for parking spaces.

While Criteria 1 and 4 were specific to The EV Project, Criteria 2 and 3 remain important in successful deployment of public infrastructure.

\section{EV Micro-Climate Planning}

\section{The Plan}

The EV Micro-Climate approach to planning involved the local EV Project representative identifying a local stakeholder advisory group to assist in identification of target infrastructure sites and solicitation of charging site hosts. The advisory group included key stakeholders who were highly motivated to support widespread adoption of PEVs in their region. Group participants included utilities, universities, PEV enthusiasts, PEV manufacturers, Clean Cities Coalitions, business development agencies, Chambers of Commerce, PEV associations, the Association of Governments, and representatives from city, county, and state governments. Included in many of the regional advisory groups were city planners with significant knowledge and experience in this type of planning effort.

The end goal of the advisory group was to publish specific goals for a long-range infrastructure plan and a near-term plan (i.e., EV Micro-Climate Plan, which encompasses the equipment to be delivered by The EV Project). Heat maps would be generated in each of the regions reflecting areas where high densities, medium densities, and low densities of EVSE infrastructure would be placed.

In these regions, the advisory groups established weighting factors for existing zoning areas, known traffic patterns, employment densities, destination attractions, and demographics of early adopters. Using multivariate analysis and geographic information systems, these data sets contributed to the mapping of the planned distribution and density of EVSE. Figure A-2 shows the density map for Portland, Oregon.

These maps would be specific enough to solicit specific charging site hosts in these regions. All regions published these "heat maps" in their respective Micro-Climate plan documents that are available at avt.inl.gov/evproject.shtml.

\section{Deployment Results}

Once the target areas for deployment had been identified, solicitation for charging site hosts in the target regions began. Reference 2 reported the challenges in enlisting charging site hosts that were common to all regions of The EV Project. Nevertheless, it also reports that $98 \%$ of the installed EVSE were within the target areas in San Diego. The region was successful in meeting the target goals of The EV Project.

\section{Utilization Comparisons}

Given that the target areas were planned for high turnover and high utilization, the next evaluation was whether there was a significant difference in the utilization of EVSE between those installed in the target regions versus those installed outside the target regions. With only $2 \%$ of the EVSE installed outside the target areas, San Diego was not a suitable region to continue the case study for this topic; therefore, Portland, Oregon was selected. This evaluation is documented in Reference 3. It reports that $74 \%$ of The EV Project's available EVSE were placed in the high 
utilization zones. EVSE placed in the high utilization zones experienced $87 \%$ greater charge events per week than those outside these zones and had average vehicle connect time periods 4.4 times longer than those outside these zones.

In the last 6 months of 2013, 7\% of all away-from-home parking events by EV Project participant PEVs in San Diego included charging. ${ }^{8}$ An average of $26 \%$ of The EV Project participants that parked near (i.e., within $300 \mathrm{ft}$ ) an EVSE installed by The EV Project actually charged at that site, whereas only $6 \%$ of participant PEVs that parked away from these project EVSE charged at some other EVSE. ${ }^{9}$

Thus, data from both San Diego and Portland documented that EVSE installed by The EV Project in target areas met key considerations significantly better than those outside.

\section{Direct Solicitation of Charging Site Hosts}

The second approach identified involved direct solicitation of charging site hosts without a focus on the key considerations noted above. This approach was used by other EVSE providers and occurred in EV Project regions when:

- Key stakeholders desired EVSE installations in other locations in response to specific interests.

- Difficulties in soliciting charging site hosts within target zones threatened meeting the project's installation deadlines.

- $\quad$ Special fleet and workplace studies were performed within The EV Project for additional analysis.

In Portland, this solicitation resulted in installations in three zones of expected high utilization, medium utilization, and low utilization. Table 1 presents the 18 lowest utilized EVSE from the start of The EV Project through August 2013 in the Portland area. ${ }^{10}$

None of these locations are involved in retail sales or services; missing Criteria 2 and 3 of the key considerations is important in the planning process for high EVSE utilization.

Decision makers in deployment of some publicly accessible EVSE may not consider Criteria 2 and 3 as important considerations that may take precedence. However, if high utilization and high turnover are important, venue selection is also important. In fact, two of the most highly utilized sites in Portland were in the medium and one was in the low expected utilization zones.
Table 1. Lowest utilized EVSE in Portland, Oregon.

\begin{tabular}{cccc}
\hline Venue & Sub-Venue & Zone & Events/Wk \\
\hline Fleet & NA & Medium & 0.04 \\
Public/Munic. & Public Works & High & 0.05 \\
Workplace & Utility & Medium & 0.10 \\
Public/Munic. & Military & Low & 0.11 \\
Public/Munic. & Senior Center & High & 0.11 \\
Workplace & Utility & Medium & 0.13 \\
Public/Munic. & Public Bldg & Low & 0.14 \\
Public/Munic. & Military & Low & 0.16 \\
Public/Munic. & Parking Lot & High & 0.16 \\
Workplace & Utility & Low & 0.18 \\
Hotel & NA & High & 0.20 \\
Public/Munic. & City Hall & High & 0.22 \\
Medical & NA & High & 0.24 \\
Workplace & Utility & Medium & 0.27 \\
Workplace & Utility & High & 0.28 \\
Public/Munic. & City Hall & High & 0.28 \\
Public/Munic. & Public Services & Medium & 0.31 \\
Hotel & NA & High & 0.38 \\
\hline
\end{tabular}

\section{Plug-In Electric Vehicle Driver Preferences in Site Selection}

As a partner in The EV Project, the University of California at Davis surveyed 461 participants in the San Diego area of The EV Project, requesting their suggestions for publicly accessible charging station locations. The EV

Micro-Climate plan for San Diego had been published before these surveys; the survey results did not impact deployment of EVSE. 4

Respondents provided 172 specific locations for AC Level 2 EVSE. Survey respondents generally provided well-reasoned locations that would be of general PEV attraction; all but a few were already targeted by the planning process. The few not planned showed very low or no actual utilization.

\section{Sites in High-Traffic Areas}

One of the planning tools used by The EV Project's stakeholder groups was traffic flows. However, this tool alone was not sufficient for meeting the key considerations of high utilization and high turnover. A high population of PEVs is helpful, but a nearby destination is required to get the PEV driver to stop and recharge. Venue selection involving destination attractions may be of higher value in selecting infrastructure sites. 


\section{Other Observations}

\section{Use of Publicly Accessible Electric Vehicle Supply Equipment}

The case study for San Diego also found that in the last 6 months of $2013,32 \%$ of project participant PEVs never charged away from home. Only $7 \%$ of all away-from-home parking included charging. This includes public and workplace charging. Even when parked near (i.e., within $300 \mathrm{ft}$ ) an installed AC Level 2 EVSE, only $26 \%$ of PEV drivers actually charged at that EVSE. In a previous survey of PEV drivers in The EV Project, $69 \%$ of respondents reported that they very rarely or never use public charging. ${ }^{11}$ It was also evident in this survey that publicly accessible EVSE needed to be "... relatively convenient to the destination of the PEV driver or along their intended route..."12 This again points to the importance of Criteria 2 and 3 in the key considerations section.

\section{Venues}

In San Diego, The EV Project's non-residential EVSE venues that attracted the most parking were workplace, retail (both shopping malls and large retail chains), and public parking lots. The venues attracting the most charging were workplace and shopping malls. ${ }^{13}$ Ninety-four of the top 100 most frequented parking locations were at venues involving retail operations. ${ }^{14}$

\section{Conclusions}

Based on experience within The EV Project, an effective installation location should include high utilization and high turnover of PEVs charging at the EVSE. An EVSE unit with low utilization is not only of little benefit to the host, but it may be a detriment to the adoption of PEVs.

The objective of gaining highly utilized EVSE is best met by advanced planning, including the site selection criteria employed by The EV Project. Local planning advisory groups can best identify target areas expected to attract PEV drivers to park and charge when they have access to zoning maps, known traffic patterns, employment densities, destination attractions, and the demographics of early adopters. Selection of a destination venue is also highly important to identify locations where PEV drivers are likely to park and can also charge when it is either convenient or needed.

\section{About The EV Project}

The EV Project was the largest PEV infrastructure demonstration project in the world, equally funded by the United States Department of Energy (DOE) through the American Recovery and Reinvestment Act and private sector partners. The EV Project deployed over 12,000 AC Level 2 charging stations for residential and commercial use and over 100 dual-port direct current fast chargers in 17 U.S. regions. Approximately 8,300 Nissan Leafs ${ }^{\mathrm{TM}}$, Chevrolet Volts, and Smart ForTwo Electric Drive vehicles were enrolled in the project.

Project participants gave written consent for EV Project researchers to collect and analyze data from their vehicles and/or charging units. Data collected from the vehicles and charging infrastructure represented almost 125 million miles of driving and 4 million charging events. The data collection phase of The EV Project ran from January 1, 2011, through December 31, 2013. Idaho National Laboratory is responsible for analyzing the data and publishing summary reports, technical papers, and lessons learned on vehicle and charging unit use.

\section{Company Profile}

Idaho National Laboratory is one of DOE's

10 multi-program national laboratories. The laboratory performs work in each of DOE's strategic goal areas: energy, national security, science, and the environment. Idaho National Laboratory is the nation's leading center for nuclear energy research and development. Day-to-day management and operation of the laboratory is the responsibility of Battelle Energy Alliance.

For more information, visit avt.inl.gov/evproject.shtml and avt.inl.gov/chargepoint.shtml.

\section{References}

${ }^{1}$ The EV Micro-Climate Planning Process, http://avt.inl.gov/pdf/EVProj/EVMicroClimatePlanningProce ssV1.0.pdf.

${ }^{2}$ How Well Did Non-Residential Electric Vehicle Supply Equipment Installations Match the Planned Areas in San Diego?, http://avt.inl.gov/pdf/EVProj/NonresEVSEInstallationvsPlan SDINLApril2015.pdf.

${ }^{3}$ How Does Utilization of Non-Residential Electric Vehicle Supply Equipment Compare Between those Installed in Oregon in Planned versus Unplanned Locations?, http://avt.inl.gov/pdf/EVProj/UtilizationOfNonResEVSEInsta llationVsPlan.pdf.

${ }^{4}$ Are Plug-In Electric Vehicle Driver Preferences an Effective Method for Siting Publicly Accessible Electric Vehicle Supply Equipment?, avt.inl.gov/evproject.shtml.

${ }^{5}$ "California Statewide Charging Survey: What Do Drivers Want?," UCD-ITSRR-13-14, Nicolas, Tal, Woodjack, Scrivano. 
${ }^{6}$ Characterize Plug-In Electric Vehicle Driver Away-FromHome Parking Behavior in San Diego, avt.inl.gov/evproject.shtml.

${ }^{7}$ Op.Cit. Installation Match.

${ }^{8}$ Op.Cit Away-from-Home Parking Behavior.

${ }^{9}$ Do Plug-In Electric Vehicle Drivers Park near Publicly Accessible Electric Vehicle Supply Equipment in San Diego but Not Use Them?, avt.inl.gov/evproject.shtml.
${ }^{10}$ Op.Cit. Utilization.

${ }^{11}$ How Do The EV Project Participants Feel About Charging Their Electric Vehicle Away From Home?,

http://avt.inl.gov/pdf/EVProj/EVProjectParticipantsAndAway FromHomeCharging.pdf.

${ }^{12}$ ibid.

${ }^{13}$ Op.Cit. PEV Drivers Park.

${ }^{14}$ Op.Cit. Characterize PEV Driver. 


\section{Appendix A Supporting Figures}

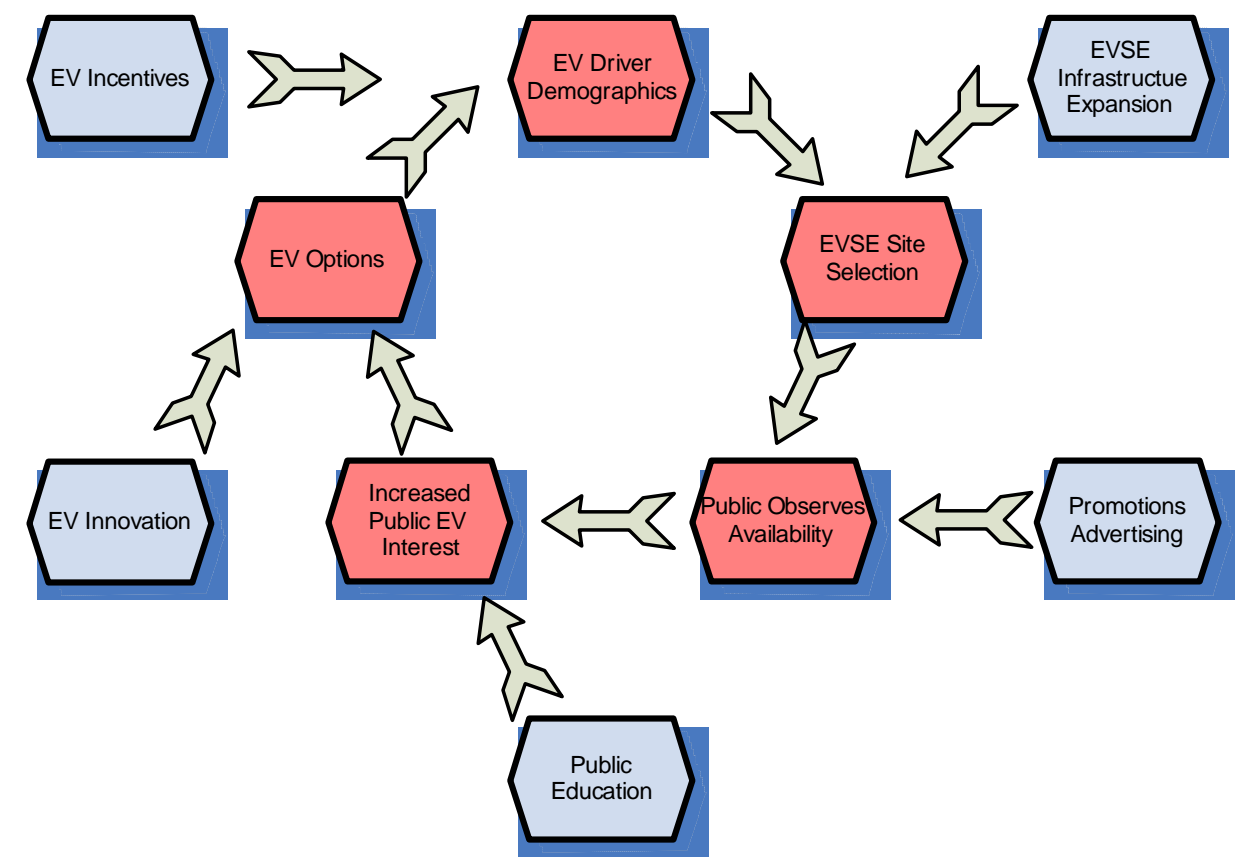

Figure A-1. PEV and EVSE promotion.

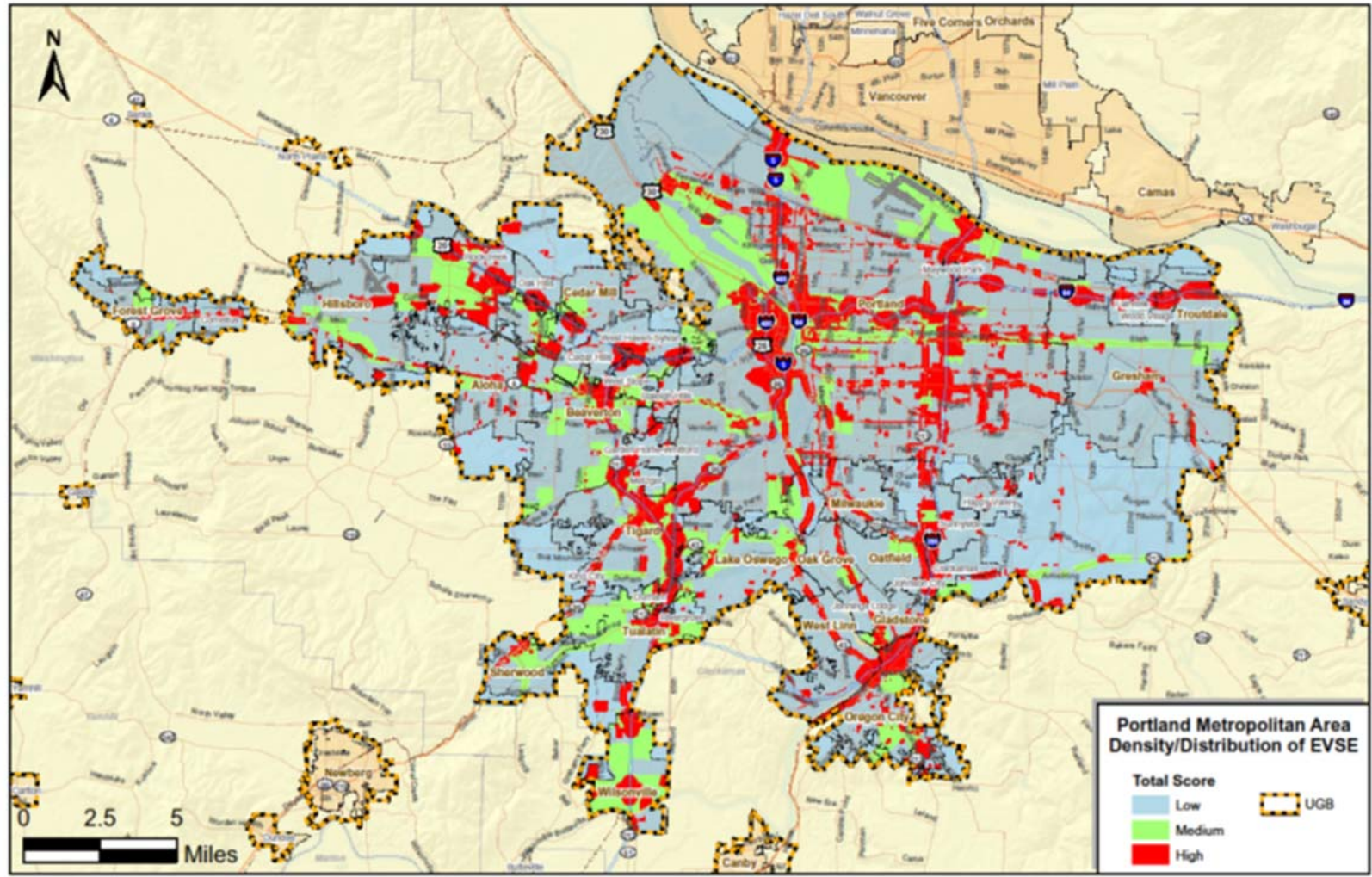

Figure A-2. EVSE infrastructure density map for Portland, Oregon. 